\title{
Dynamic Allocation of STBC for OFDM based on WSSUS Channel Model
}

\author{
F. Meucci ${ }^{1,2}$, L.Pierucci ${ }^{1}$, E. Del $\operatorname{Re}^{1}$, R. Prasad ${ }^{2}$ \\ ${ }^{1}$ University of Florence, Florence, Italy \\ ${ }^{2}$ Center for TeleInFrastruktur (CTIF), Aalborg University, Aalborg, Denmark
}

\begin{abstract}
In this paper we analyze the effect of the frequency selectivity and time variation of the wireless channel over Space Time Block Code (STBC)s in an Orthogonal Frequency Division Multiplexing (OFDM) system for a mobile User Terminal (UT). In order to mitigate the influence of the channel variability, we propose a dynamic allocation over the time-frequency OFDM grid: a criterion for dynamic allocation direction is derived for a Wide Sense Stationary - Uncorrelated Scattering (WSSUS) channel. The dynamic allocation is assisted by a feedback augmented only by one-bit.

Results shows that mobile users can greatly improve Bit Error Rate (BER) performance with dynamically allocated symbols. The Symbol Error Probability (SEP) gain is analytically studied for the Alamouti code and computer simulations are provided for ITU Vehicular channel model for a Downlink (DL) connection in a IEEE802.16e system.

Index Terms-Wireless Wide Area Networks, Mobility Management, Dynamic Allocation, STBC, OFDM.
\end{abstract}

\section{INTRODUCTION}

In the future of the wireless communication systems the traffic directed to the UTs is growing with very fast pace. Multimedia streams, double and triple play[1], Internet browsing, file transfers are requiring high capacity links. The scarcity of spectrum resource is thus forcing wireless communication engineers to push for high spectral efficiencies.

When the UTs are mobile, the channel has time variability due to the Doppler effect which is directly proportional to the carrier frequency. In order to have larger spectrum allocated, the carrier frequencies tend to move up thus worsening the Doppler effect. Next generation systems are aiming to serve UT with real-time connections, such as Voice over IP (VoIP), with mobility up to $300 \mathrm{Km} / \mathrm{h}$ (high velocity trains). For these reasons, transmission techniques aiming at high spectral efficiencies in mobile channels will be of fundamental importance.

Multiple antennas systems, Multiple Input Multiple Output (MIMO), have added a new resource dimension to be exploited. One way to utilize the antenna resource and to leverage capacity is to provide the receiver with diversity, i.e. with multiple copies of the transmitted signal in order to combat the inherent fading of the radio link. Space Time Code (STC) are a promising paradigm to exploit the multi-antenna resource, especially STBC due to their simple linear decoding at the receiver [2], [3]. The first STBC code was proposed by Alamouti, [4]. Two transmitting antennas are providing a diversity order of $2 \times n$, where $n$ is the number of the receiving antennas. In the case of one receiving antenna, a maximum diversity order of 2 can be achieved: the same performance of a Maximum Ratio Combining (MRC) scheme with two diversity branches, thus moving the problem of multiple RF chains from the UT to the Base Station (BS).

In OFDM systems, an additional degree of freedom can be exploited. The ST coding can be expanded to SpaceTime-Frequency (STF) coding. It has been shown, in [5], that space-frequency-only coded OFDM systems cannot achieve full diversity: coding over the three STF dimensions is needed. Three dimension coding has very complex implementation and simpler STF solutions have been proposed in literature. The OFDM subcarriers frequency decorrelation can be exploited in order to preserve (and enhance) the diversity when the channel spatial correlation is high (thus the channel matrix rank is low). This can be accomplished using subcarriers grouping as in [6], [7] or multiple channel fading blocks as proposed in [8]. In [7], a constellation precoding and Space-Time (ST) component coding is used. These works do not deal with the problem of channel variations due to mobility but considered only the frequency domain.

The STBC are based on the assumption that the channel remains constant over multiple signaling time slots; these codes provide a diversity enhanced signal at the receiver which can be decoded with simple linear operations. In [3], [4], the channel is assumed to be constant over the time length of the code. However, this assumption is usually unverified especially when the code length is quite long and UTs are moving. In this case, the channel value at time $t$ can be expressed in relation to the preceding one $(t-1)$ with a correlation coefficient $\rho<1$ : the SEP have been derived for the Alamouti scheme as function of $\rho,[9]$.

In this work, we assume channel spatial decorrelation and we aim to enhance the STBC performance with highly mobile UTs. The UT is assumed to move at fixed velocity. The movement of the UT causes variations in the frequency domain due to varying multi-path delay spread and in the time domain due to the Doppler effect.

We propose a dynamic allocation of the STBC data symbols along the time or frequency direction with a singlebit feedback aiming to maximize the correlation between successive STBC symbols. We show that existing STBCs can maintain the diversity if dynamically allocated. We study the effect of the channel multi-path fading and Doppler spectrum over STBCs in an OFDM system. An OFDM-based system has good performance in multi-path environments and great allocation flexibility, [10]. The dynamic allocation of STBC 
here proposed has low impact on the legacy OFDM systems already developed.

Based on WSSUS channel model [11], we derive the correlation coefficients among subcarriers, $\rho_{f}(\Delta f)$, and between different signaling times, $\rho_{t}(\Delta t)$. The correlation coefficients are used to analytically calculate the SEP expression for the Alamouti-coded symbols. Based on which correlation coefficient is the highest between $\rho_{t}$ and $\rho_{f}$, the symbols are allocated, tracking the variation in channel PDP and terminal velocity. Antennas are considered uncorrelated both at the UT and at the BS.

In Section II the OFDM system model is explained. In Section III, the channel model is presented: correlation coefficients are derived based on Power Delay Profile (PDP) and terminal velocity with various Doppler spectrum models. After, in Section IV, the method for calculating closed-form SEP for correlated channel is reported for the case of Alamouti scheme. The proposed dynamic allocation with feedback is presented in Section V and then results are shown in terms of BER gains. Sec. VI concludes the paper and reports current work in progress.

\section{System MODEL}

In OFDM systems, the data to be transmitted is organized in frames which can be represented as a grid or a matrix with $N_{\text {data }} \times N_{\text {ofdm }}$ entries, [12], where $N_{\text {data }}$ is the number of data subcarrier per OFDM symbol and $N_{o f d m}$ is the number of OFDM symbols in one frame. If we consider a system with $m$ transmitting and $n$ receiving antennas, a frame is composed for each antenna, leading to $m$ frames available for symbol allocation. The OFDM symbol duration in time, later indicated with $T_{o f d m}$, and the subcarrier spacing in frequency, $\Delta F$, are fundamental for calculating the STBC BER performance since they represent the minimum distance, thus maximum channel correlation, that the symbols can experience once allocated on the OFDM grid, [12].

Fig. 1 shows a diagram of a MIMO-OFDM system with a generic STBC encoder. The symbols from the source are first modulated and then enter the STBC encoder. The number of the outputs of the encoder is equal to $m$, the number of transmitting antennas. For each of these output, a mapping function places the coded symbols on the frame based on a single-bit feedback from the UT.

In Fig. 2 we plot the channel magnitude $\left(\left\|\mathbf{H}_{i j}\right\|-[d B]\right)$ of one frame in the frequency domain. The channel model is the ITU Vehicular Channel Type A with a terminal velocity of $100 \mathrm{Km} / \mathrm{h}$. From the plot, one can easily follow the channel frequency profile and its variation over time due to UT mobility. When MIMO is used, a two-dimensional channel transfer function is measured for each couple of transmittingreceiving antennas.

\section{WSSUS CHANNEL: THE TIME-FREQUENCY} CORRELATION FunCTION IN THE FREQUENCY DOMAIN

In order to asset a generalized criterion to decide in which direction the allocation of the STBC symbols should be

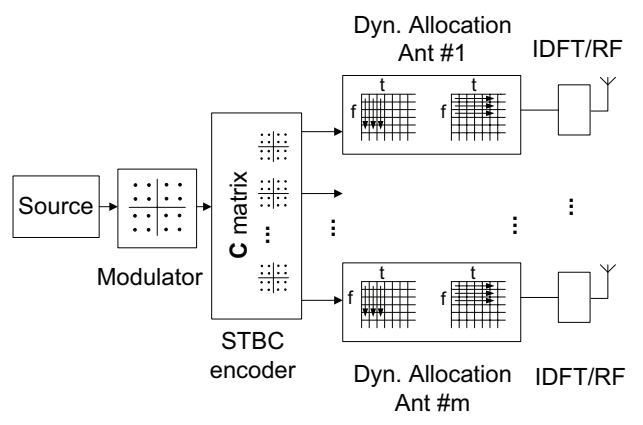

Fig. 1. MIMO-OFDM system model with STBC encoder

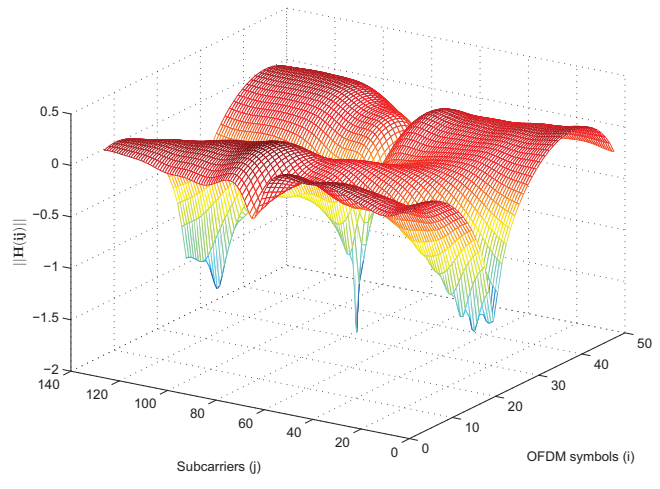

Fig. 2. OFDM channel grid, 128 subcarriers and 48 OFDM symbols.

performed, we need to model the correlation coefficients $\rho_{t}$ and $\rho_{f}$ between subcarriers both along time and frequency direction respectively. In Sec. IV, we compute the BEP of the Alamouti scheme for different correlation coefficients which are obtained based on the derivation of this section.

Almost in all the practical cases, the radio channel behavior can be effectively described as a Linear Time Variant (LTV) system. Stemming from the nomenclature used in [11], where a set of dual frequency-time functions where defined for LTV systems, we study the performance of diversity schemes depending on the spaced-frequency, spaced-time correlation function: $\phi_{H}(\Delta t, \Delta f)$.

From the time-variant channel impulse response, $h(t, \tau)$, the time-variant channel transfer function in the Frequency Domain (FD), $H(f, t)$, can be expressed via a the Fourier transform. The channel matrix $\mathbf{H}(i, j)\left(h_{i j} \in \mathbb{C}\right)$ is obtained sampling the value of $H(f, t)$ at $f=f_{0}+\Delta F \cdot i$ where $f_{0}$ is the lowest frequency subcarrier and at $t=T_{0}+T_{o f d m} \cdot j$.

The channel correlation function in the FD is:

$$
\phi_{H}\left(f_{1}, f_{2}, t_{1}, t_{2}\right)=E\left\{H^{*}\left(f_{1}, t_{1}\right) H\left(f_{2}, t_{2}\right)\right\}
$$

Under the assumption that the channel is Wide Sense Stationary (WSS), the correlation function is depending only on time and frequency differences, thus Eq. 1 can be written as:

$$
\phi_{H}(\Delta t, \Delta f)=E\left\{H^{*}(f, t) H(f+\Delta f, t+\Delta t)\right\}
$$


which is the spaced-frequency, spaced-time correlation function in the frequency domain.

The real value of $\phi_{H}(\Delta t, \Delta f)$, sampled at multiplies of $\Delta F$ and $T_{o f d m}$, represents the correlation between various $\mathbf{h}_{i j}$ : $\phi_{H}(i, j)$. The correlation along time or frequency direction on the OFDMA grid can be obtain as:

$$
\begin{aligned}
\rho_{f}(i) & =\left.\rho_{f}(\Delta f)\right|_{\Delta f=f_{0}+i \cdot \Delta F} \\
\rho_{t}(j) & =\left.\rho_{t}(\Delta t)\right|_{\Delta t=T_{0}+j \cdot T_{o f d m}}
\end{aligned}
$$

The minimum time and frequency separations are lower bounded by the OFDM system design parameters, respectively $T_{o f d m}$ and $\Delta F$.

The functions $\phi_{H}(\Delta f)$ and $\phi_{H}(\Delta t)$ can be obtained as the Fourier transforms of the PDP $\phi_{h}(\tau)$ and of the Doppler Spectrum $S_{H}(\nu)$ (where $\nu$ is the Doppler shift variable), respectively. Through the estimation of the PDP and of the Doppler Spectrum, the proposed dynamic allocation can minimize the BER for the STBCs.

In OFDM systems, the Root Mean Square (RMS) delay spread $\tau_{r m s}$ and the Doppler frequency can be easily estimated. Various methods have been proposed in literature: [13], [14], [15], [16]. When only the $\tau_{r m s}$ and $f_{d}$ are available, an assumption about the shape of the PDP and of the Doppler Spectrum are needed.

If an exponential decaying PDP model is assumed, the related spaced-frequency correlation function can be expressed in function of the $\tau_{r m s}$ as:

$$
\phi_{H}(\Delta f)=\frac{1}{1+j 2 \pi \tau_{r m s} \Delta f}
$$

The frequency correlation $\left(\rho_{f}\right)$ is illustrated in Fig. 3. The PDP is assumed as in Eq. 4 and various correlation are reported for different $\tau_{r m s}$ and subcarriers spacing.

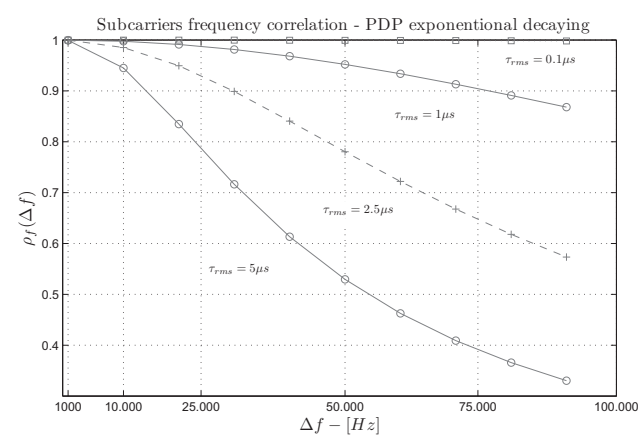

Fig. 3. Expontential Deacying PDP - subcarriers correlation over frequency separation for various $\tau_{r m s}$ delays.

The spaced-time correlation function in the time domain is obtained from $\phi_{H}(\Delta t, \Delta f)$ setting the $\Delta f=0$ or alternatively operating a back Fourier transform on the Doppler power spectrum $S_{H}(\nu)$, from the shift variable $\nu \rightarrow \delta t$ The Doppler spectrum depends on the geometrical assumption made on the direction of the multipaths impinging on the receiving antenna. The most common model assumed is the Jakes model [17]. This model is between the two extreme cases of two rays and uniform distribution of the arriving rays at the receiving antennas.

The time correlation is shown for Jakes spectrum in Fig. 4. The Doppler frequencies shown are corresponding to UT velocities of $60-125-250 \mathrm{Km} / \mathrm{h}$ at a carrier frequency of $6 \mathrm{GHz}$. When $T_{\text {ofdm }}=100 \mu \mathrm{s}$ the correlation coefficient ranges from 1 to 0.8 .

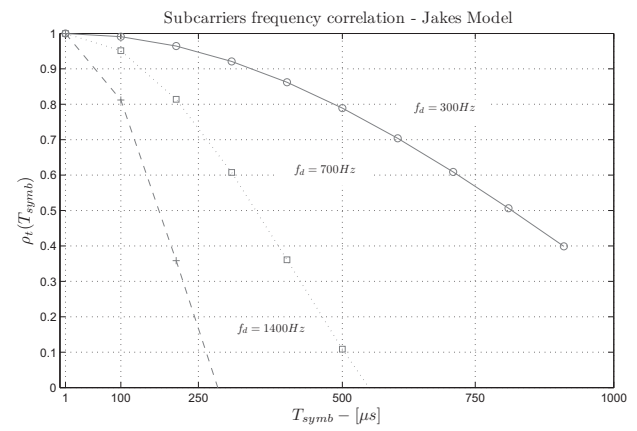

Fig. 4. Jakes model - subcarriers correlation over time for various Doppler frequencies.

Comparing the values of $\rho_{t}$ and $\rho_{f}$ from Figs. 3- 4, we note that the correlation coefficient can be lower in the time or frequency domain, depending on the relative ratio of terminal velocity and delay spread. Thus, the BER performance of STBCs can be enhanced if a dynamic allocation direction is implemented as shown in Sec. V.

\section{Diversity SCHEMES In UNCORRELATED CHANNELS}

A space-time block code is defined by a coding matrix which maps constellation symbols over the $m$ transmitting antennas in successive $T$ time slots. The coding matrix $\mathbf{C}$ has to be orthogonal since a non-orthogonal $\mathbf{C}$ will result in Inter Subcarrier Interference (ISI) interference at the receiver. Orthogonal designs for real and complex constellation symbols has been derived in [2] based on the orthogonal theory by Radon and Hurwitz. Matrix $\mathbf{C} \in \mathbb{R}^{L \times m}$ and maps $L \times m$ data symbols over successive $L$ time slots over the transmitting antennas. The decoding process for the ST codes is simple and it is based on linear combination of the transmitted signals from each of the $m$ transmitting antennas.

The first and simplest STBC was proposed in [4], where $m=2$ both for the case of $n=1$ and $n=2$. The coding matrix $\mathbf{C}$ can be written as:

$$
\mathbf{C}_{2}=\left[\begin{array}{cc}
s_{1} & s_{2} \\
-s_{2}^{*} & s_{1}^{*}
\end{array}\right]
$$

STBCs assume that the channel remains constant during the time duration of the code ( $L$ signaling time slots). Alamouti code implies that the channel is constant over 2 signaling period. The constant channel assumption is easily not verified particularly in quick time variant channels.

When channel coefficient are not correlated $(\rho \neq 1)$, the BER performance of the STBCs is influenced by the correlation coefficients between two consecutive symbols: $\rho_{f}$ if symbols are allocated along frequency, $\rho_{t}$ otherwise. 
The SEP performance for the Alamouti transmit diversity scheme with a time-varying channel has been derived for narrow-band partially correlated Rayleigh channels for BPSK. We can express the channel coefficients accounting the correlation as:

$$
\begin{aligned}
& h_{1}^{1}=\rho h_{1}^{2}+\sqrt{1-\rho^{2}} \epsilon_{1} \\
& h_{2}^{2}=\rho h_{2}^{1}+\sqrt{1-\rho^{2}} \epsilon_{2}
\end{aligned}
$$

where the superscript indicate the successive channel coefficient (either in the time or frequency direction of the OFDM grid) and the subscript indicates the number of the transmit antenna. The random variables $\epsilon_{i}$ are distributed with Rayleigh distribution as the channel coefficients. If the correlation $\rho=1$, the transmit diversity scheme is not suffering any performance losses. The other extreme case is represented by $\rho=0$, when no correlation exists between two successive channel coefficients.

When $\rho$ is comprised between these two extremes, the SEP for a Zero Forcing (ZF) detector with M-QAM modulation and coherent detection can be expressed based on the SNR distribution at the output of the STBC combiner: $p_{\gamma}(\gamma)=$ $\frac{2\left(1-\rho^{2}\right)}{\bar{\gamma}} e^{2 \gamma / \bar{\gamma}}+4 \rho^{2} \frac{\gamma}{\gamma} e^{2 \gamma / \bar{\gamma}},[9]$.

To obtain the SEP over the fading channel, the SEP conditioned on the instantaneous SNR needs to be integrated over the SNR distribution. Following the approach in [18],

$$
S E P_{M}(\bar{\gamma})=4 K I^{(1)}(a(M), \gamma)-4 K^{2} I^{(2)}(a(M), \gamma)
$$

where $K=(\sqrt{M}-1) / \sqrt{M}, M$ is the modulation order, $a^{2}=3 \log _{2}(M) /(M-1)$ and

$$
\begin{aligned}
& I^{(1)}(a(M), \gamma)=1 / \pi \int_{0}^{\pi / 2} M_{\gamma}(-s(\theta)) d \theta \\
& I^{(2)}(a(M), \gamma)=1 / \pi \int_{0}^{\pi / 4} M_{\gamma}(-s(\theta)) d \theta
\end{aligned}
$$

In the integrand functions: $s=\frac{a^{2}}{2 \sin ^{2}(\theta)}$ and $M_{\gamma}(s)$ is the characteristic function of the SNR distribution at the output of the ZF receiver: $M_{\gamma}(s)=\frac{\left(1-\rho^{2}\right)}{(s \bar{\gamma} / 2+1)}+\frac{\rho^{2}}{(s \bar{\gamma} / 2+1)^{2}}$.

In Fig. 5, analytical SEP curves are reported for 4-1664 QAM modulations with correlation coefficients $\rho \in$ $[1,0.9,08]$. For SNR levels above $4 d B$, the Alamouti scheme is very sensitive to channel variability and the performance is highly degraded. For a 4-QAM modulation at $S N R=20 \mathrm{~dB}$, the SEP shifts from around $10^{-4}$ to more then $3 \cdot 10^{-3}$.

\section{DYNAMIC DIRECTION ALLOCATION}

In the previous Sec. III we have analyzed the correlation coefficient along time and frequency allocation direction based on the spaced-time, spaced-frequency correlation function in the frequency domain. Then, based on general correlation $\rho$, SEP has been expressed in closed-form for the Alamouti scheme in Sec. IV.

In this section we propose the adaptive allocation on the OFDM grid based on the estimated delay spread $\tau_{r m s}$ and

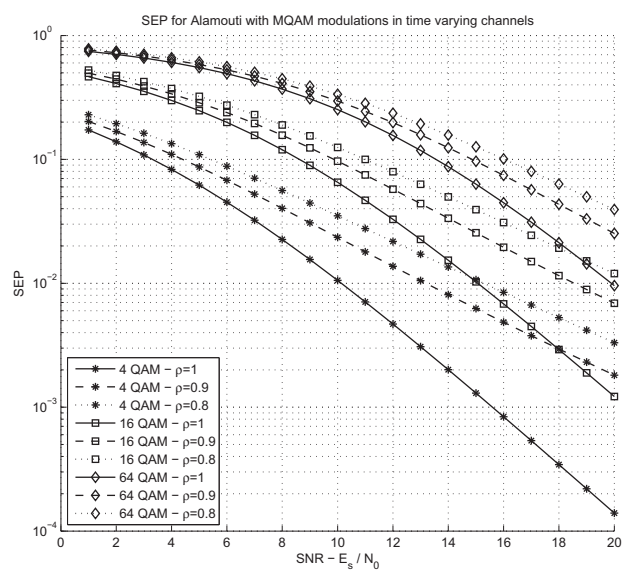

Fig. 5. Symbol Error Probability for Alamouti scheme for 4-16-64 QAM modulations over narrow-band partially correlated Rayleigh channel.

Doppler frequency $f_{d}$. We assume a perfect channel estimation. In Tab. I we report the OFDM system settings which refer to IEEE standard 802.16e.

TABLE I

OFDM SYSTEM PARAMETERS, IEEE802.16E STANDARD

\begin{tabular}{||l|l|l||}
\hline Parameter & Description & Value in $802.16 \mathrm{e}$ \\
\hline$c_{f}$ & Carrier frequency & $2.5 \mathrm{GHz}$ \\
\hline $\mathrm{BW}$ & Total bandwidth & $10 \mathrm{MHz}$ \\
\hline$N_{f f t}$ & Number of FFT points & 1024 \\
\hline$\Delta f$ & Subcarrier spacing & $10.9375 \mathrm{kHz}$ \\
\hline$T_{o f d m}$ & OFDM symbol duration without CP & $91.43 \mathrm{us}$ \\
\hline $\mathrm{CP}$ & Cyclic prefix length & $1 / 8 T_{o f d m}$ \\
\hline$T_{o f d m}^{C P}$ & symbol duration with CP & $102.86 \mathrm{us}$ \\
\hline $\mathrm{F}$ & Frame length & $5 \mathrm{~ms}$ \\
\hline $\mathrm{N}$ & Number of OFDM symbols in frame & 47 \\
\hline
\end{tabular}

The proposed system is illustrated in Fig. 6. The system calculates $\rho_{t}$ and $\rho_{f}$. Then it decides the allocation direction where the correlation is higher. These two coefficients can be exactly calculated if the PDP and the Doppler Spectrum of the channel are known. However, assumptions can be made on the Doppler Spectrum and PDP shapes. In this case, the system needs only to estimate the channel delay spread and the Doppler frequency.

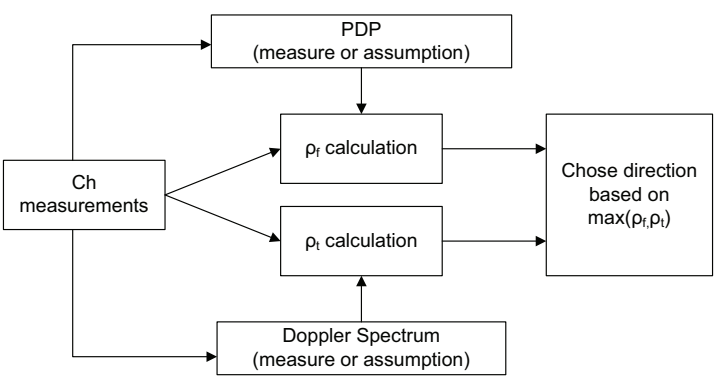

Fig. 6. The dynamic allocation direction OFDM system.

The computer simulations show the SER performance of the OFDM system with the Alamouti scheme in a DL con- 


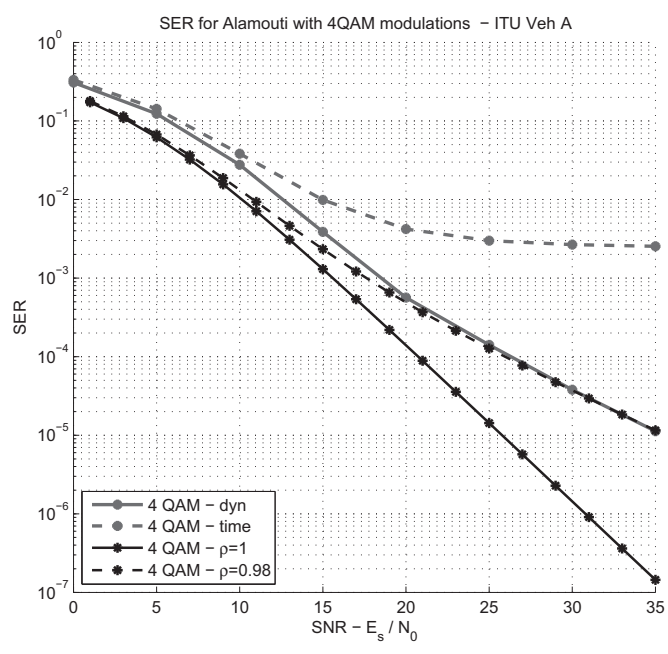

Fig. 7. BER improvement for ITU Vehicular Channel model A with UT at $150 \mathrm{Km} / \mathrm{h}$

nection to a high velocity UT averaged over 10 seconds. The dynamic allocation operates with the assumption of Jakes Doppler spectrum and Exponential decaying PDP. The ITU vehicular channel model $\mathrm{A}$ has a $\tau_{r m s}=3.32 \mu \mathrm{s}$. In the case of the exponential decaying PDP, we can calculate the expected correlation between adjacent subcarriers along the frequency direction obtaining $\rho_{f}=\phi_{H}(\Delta F) \approx 0.98$, $\Delta F=10.9375 \mathrm{kHz}$.

In Fig. 7, we can see that the dynamic allocation direction preserves the diversity order of the Alamouti scheme. The SER of the system tends to follow the theoretical one calculated along the frequency domain with the corresponding correlation coefficient. The correlation coefficient along the time direction $\left(\rho_{t}\right)$ is generally lower than $\rho_{f}$ and the dynamic proposed system optimizes the allocation in order to obtain the minimum SER. The difference between the theoretical and the simulated curves originates from the InterCarrier Interference (ICI) of the OFDM system, since the theoretical calculation have been derived under the assumption of narrow-band channel.

On the other hand, in the case of receivers with very low Doppler frequencies (for example fixed receivers which generally show an $f_{d}$ of the order of some units of Hertz, due to moving object in the radio environment) the symbols would be mainly allocated along the time direction. This provides better SER performance respect to a fixed allocation along the frequency direction since the frequency profile could have very low correlation due to high delay spread.

\section{CONCLUSION AND Future WORK}

The system proposed is able to adapt the allocation direction on the OFDM grid in order to best match to the channel characteristics of the user. Current standards (as IEEE802.16e) are using fixed allocation direction and they may lose the diversity enhancement of the STBC. The proposed dynamic allocation requires low modifications in the air-interface design and preserves the STBC performance which otherwise would be lost due to the terminal mobility. The feedback needs only a single-bit feedback, thus resulting in an almost negligible overhead in the network.

The strategy has been simulated implementing an Alamouti scheme, without a loss of generality. The results will be extended to a general $L$ length STBC where the improvements will show even greater enhancements, considered that the assumption of quasi-static channel is more difficult to be verified when the STBC length increases. Current work is also focusing on the statistical description of the ICI effect of the dynamic allocation proposed.

\section{REFERENCES}

[1] K. Moerman, J. Fishburn, M. Lasserre, and D. Ginsburg, "Utah's UTOPIA: an ethernet-based MPLS/VPLS triple play deployment," Communications Magazine, IEEE, vol. 43, no. 11, pp. 142-150, Nov. 2005.

[2] V. Tarokh, H. Jafarkhani, and A. Calderbank, "Space-time block codes from orthogonal designs," IEEE Trans. Inf. Theory, vol. 45, no. 5, pp. 1456-1467, July 1999.

[3] — "Space-time block coding for wireless communications: performance results," IEEE J. Sel. Areas Commun., vol. 17, no. 3, pp. 451-460, March 1999.

[4] S. Alamouti, "A simple transmit diversity technique for wireless communications," Selected Areas in Communications, IEEE Journal on, vol. 16 , no. 8, pp. 1451-1458, Oct 1998.

[5] H. Bolcskei, M. Borgmann, and A. Paulraj, "Impact of the propagation environment on the performance of space-frequency coded MIMOOFDM," IEEE J. Sel. Areas Commun., vol. 21, no. 3, pp. 427-439, April 2003.

[6] A. Molisch, M. Win, and J. Winters, "Space-time-frequency (STF) coding for MIMO-OFDM systems," IEEE Commun. Lett., vol. 6, no. 9, pp. 370-372, Sept. 2002

[7] Z. Liu, Y. Xin, and G. Giannakis, "Space-time-frequency coded OFDM over frequency-selective fading channels," IEEE Trans. Signal Process. vol. 50, no. 10, pp. 2465-2476, Oct. 2002.

[8] W. Zhang, X.-G. Xia, and P. C. Ching, "High-Rate Full-Diversity Space\&ime\&requency Codes for Broadband MIMO Block-Fading Channels," IEEE Trans. Commun., vol. 55, no. 1, pp. 25-34, Jan. 2007.

[9] A. Vielmon, Y. Li, and J. Barry, "Performance of alamouti transmit diversity over time-varying rayleigh-fading channels," IEEE Trans. Wireless Commun., vol. 3, no. 5, pp. 1369-1373, Sept. 2004.

[10] F. Meucci, A. Mihovska, B. Anggorojati, and N. Prasad, "Joint Resource Allocation and Admission Control Mechanism for an OFDMA-Based System," in Proc. of WPMC '08 to be held in Lapland, Finland, in September 8-11, 2008, 2008.

[11] P. Bello, "Characterization of randomly time-variant linear channels," IEEE transactions on Communications Systems, vol. 11, no. 4, pp. 360393, December 1963.

[12] R. van Nee and R. Prasad, OFDM for Wireless Multimedia Communi cations. Norwood, MA, USA: Artech House, Inc., 2000.

[13] K. Ramasubramanian and K. Baum, "An OFDM timing recovery scheme with inherent delay-spread estimation," in Proc. IEEE Global Telecommunications Conference GLOBECOM '01, vol. 5, 25-29 Nov. 2001, pp. 3111-3115.

[14] J.-H. Wen, S.-H. Lee, G.-R. Lee, and J.-T. Chang, "Timing and delay spread estimation scheme in OFDM systems," IEEE Trans. Consum. Electron., vol. 54, no. 2, pp. 316-320, May 2008.

[15] K. Witrisal, Y.-H. Kim, and R. Prasad, "A new method to measure parameters of frequency-selective radio channels using power measurements," IEEE Trans. Commun., vol. 49, no. 10, pp. 1788-1800, Oct. 2001.

[16] T. Yucek and H. Arslan, "Time Dispersion and Delay Spread Estimation for Adaptive OFDM Systems," IEEE Trans. Veh. Technol., vol. 57, no. 3 pp. 1715-1722, May 2008

[17] P. Dent, G. Bottomley, and T. Croft, "Jakes fading model revisited," Electronics Letters, vol. 29, no. 13, pp. 1162-1163, 24 June 1993.

[18] M. K. Simon and M.-S. Alouini, Digital Communication Over Fading Channels. Wiley-Interscience, 2005. 